\title{
Distributed Cooperative Processing and Control over Wireless Sensor Networks
}

\author{
C. Fischione, F. Graziosi, K. H. Johansson, F. Santucci ${ }^{\text {* }}$
}

\begin{abstract}
An overview of some recent advances in distributed information processing for control over wireless sensor networks is presented in this paper. Firstly, a taxonomy of fundamental control and communication schemes for these systems is introduced. Next, specific research issues are proposed and discussed with three prominent examples on distributed source coding with packet aggregation, distributed cooperative diversity and distributed cooperative localization. In regard to these examples, it is argued about some open research problems and suggestions for further investigations on joint control and communication design for distributed processing and control over wireless sensor networks.
\end{abstract}

Index Terms: Wireless Sensor Networks, Distributed Cooperation, Distributed Control, Network Control

\section{INTRODUCTION}

Real-time decision-making based on information from wireless sensor networks (WSNs) relies on many subsystems and components working together in an efficient and trustworthy manner. Despite major recent advances in hardware and software technologies, the full benefits of these control systems based on wireless sensors and actuators are still to be explored. New collaborative and adaptive algorithms are needed to enable autonomous and optimal operation. In particular, the limited amount of resources of the wireless nodes has a significant impact on all aspects of the overall system, from the amount of information that a node can process, over suitable low-attention control algorithms, to proper wireless commu-

${ }^{*}$ Work done in the framework of the HYCON Network of Exellence, contract number FP6-IST-511368. The work by C. Fischione and K. H. Johansson was partially funded also by the Swedish Foundation for Strategic Research through an Individual Grant for the Advancement of Research Leaders and by the Swedish Research Council.

${ }^{\dagger}$ C. Fischione and K. H. Johansson are with Automatic Control Lab, School of Electrical Engineering, Royal Institute of Techonolgy, Osquldas väg 10, 10044 Stockholm, Sweden. Email: $\quad$ carlofi|kallej\}eee.kth.se. F. Graziosi and F. Santucci are with DEWS Centre of Excellence of the University of L'Aquila, 67040 Poggio di Roio, Italy. E-mail: \{graziosi|santucci\}@ing.univaq.it.

Permission to make digital or hard copies of all or part of this work for personal or classroom use is granted without fee provided that copies are not made or distributed for profit or commercial advantage and that copies bear this notice and the full citation on the first page. To copy otherwise, to republish, to post on servers or to redistribute to lists, requires prior specific permission and/or a fee.

IWCMC'06, July 3-6, 2006, Vancouver, British Columbia, Canada. Copyright 2006 ACM 1-59593-306-9/06/0007 ...\$5.00. nication. Significant performance improvements can be achieved by exploiting the advantages offered by joint design of distributed collaborative processing and control over WSNs.

Collaborative processing, in the context of cellular radio system and ad hoc wireless networks, has been an area of intense research, e.g., [1]. For WSNs, however, constraints on power, memory, information processing etc., make the extension of traditional processing techniques rather challenging. In many sensing and control applications, cross-layer mechanisms should also be taken into account [2]. There are several emerging applications that are putting completely new demands on the communication systems. Examples include the coordination of groups of unmanned autonomous vehicles, which have on-board computing capabilities and communicate over wireless networks, e.g., [3]. The main goal for these systems is to collectively perform useful tasks such as reaching a pre-specified destination, gathering data or search for objects. These monitoring and control applications call for the development of decentralized schemes with self-organization capabilities. A more general set of applications are found in the area of networked embedded systems with major potentials in a wide set of areas such as automation, automotive and process industries.

The design problem should pose a trade-off between the use of the wireless communication network and the control performance. Important communication parameters include not only network throughput, but also time delay and packet loss probability. Some recent progress on control using WSNs was reported by Goldsmith et al. [2]. Specifically, it was shown that communication parameters need to be properly chosen in order to optimize the performance of control systems with feedback loops closed over wireless communication links. This means that it is important both to develop robust control techniques and to modify link layer designs when controllers cannot compensate for the performance degradations due to packet losses. It was shown in [4] that the optimization requires a cross-layer design framework, which was illustrated by a cross-layer optimization of the link layer, MAC layer, and sample period selection in the case of a networked control system. Despite these recent contributions, wireless communication in estimation and control is still an emerging and not yet mature area of research [5]. While problem-specific techniques need obviously to be developed to proficiently address the different cases, there is an underlying body of theoretical problems and technological challenges which are common, and which would greatly benefit from a unified approach.

The aim of this paper is to provide a discussion on some fundamental aspects of distributed cooperative processing and control over WSNs. The basis for the presentation is the ongoing investigations within the framework of the European Network of Excellence HYCON [6]. After a general discussion on the relevant configurations of collaborative distributed processing and control, we report on some illustrative examples, namely, distributed source cod- 


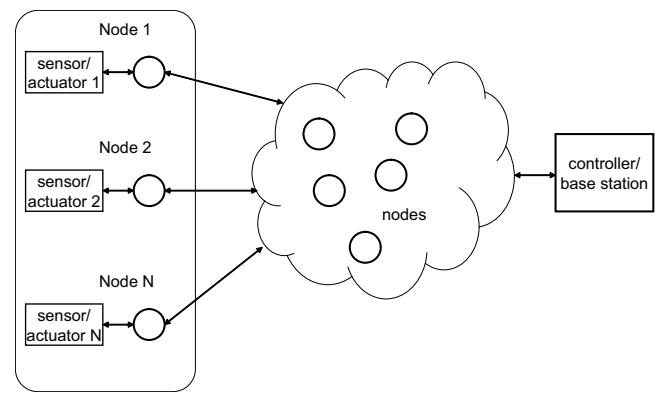

Figure 1: The distributed cooperative processing is implemented among the sensors/actuators, while the control is centralized.

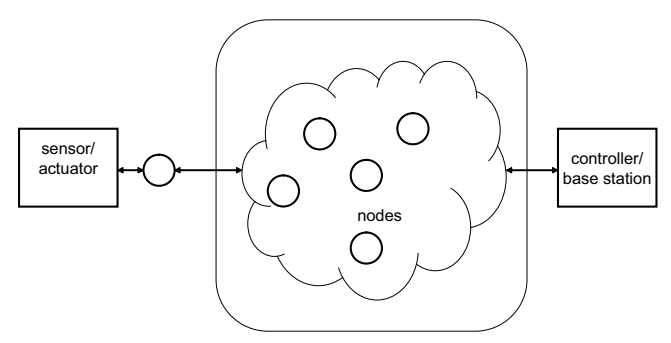

Figure 2: The distributed cooperative processing is implemented in the network, while the control is centralized.

ing and packet aggregation [7], cooperative distributed diversity [8] and cooperative distributed localization [9]. The organization of the paper is then as follows. In Section II, some relevant schemes for distributed processing and control are discussed and fundamental design constraints are evidenced. In Section III, the illustrative examples are proposed. Finally, in Section IV conclusions are given.

\section{DISTRIBUTED COOPERATIVE PROCESS- ING AND CONTROL}

Let us consider a system where wireless sensors, controllers and actuators interact for monitoring and control purposes. In the system, there are power-constrained nodes equipped with computational and wireless transmission capabilities. Sensors and actuators connect the system to one or more plants. A sensor/actuator may either have computational and communication capabilities or is directly connected to a node that has such capabilities. There are also controllers, which are components in charge of the control of the plant or the nodes.

The distributed cooperative processing may happen in several stages of the system, and may involve both communication and control aspects. We believe that the three configurations in Figs. 13 deserve particular attention.

In Fig. 1, a controller/base station receives information from the plant through the WSN. The group of peripheral sensors implements the distributed and cooperative processing, while the control is centralized. In this case, the network should allow for the delivery of information from the sensors to the controller and then send the corresponding control action to the actuators. When using this scheme, significant delays are potentially introduced in the network: communication delays (due to sensor and control signals transmitted over the network) and computation delays (induced by the computations in the nodes). The synchronization of the signal coming from the different plant plays also an important role.

With regard to the scheme of Fig. 2, a relevant feature is that there is only one sensor, but it is connected through several nodes to the controller. In this case, the distributed and cooperative process-

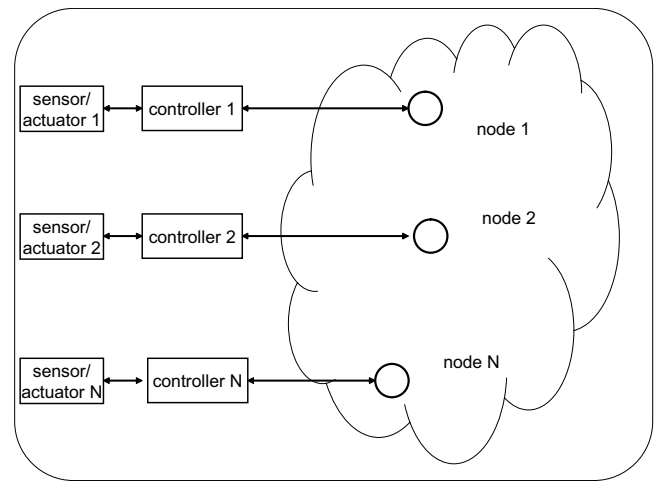

Figure 3: The distributed cooperative processing is implemented over all the network, the controllers, and the sensors/actuators.

ing takes place among the nodes of the network. The stability of the closed-loop control system may require rates of signal disconnections smaller than a certain threshold, that depends on the sampling frequency of the sensors and actuators as well as the dynamics of the plant.

A more general case is shown in Fig. 3, where the control is distributed across several control nodes, and it is assumed that the controller $i$ can use sensor readings from sensor $j \neq i$. Here distributed cooperation is supposed to happen both for the control and the communication tasks. This is thus the most challenging configuration. The controllers may need information from all the nodes of the networks, so that packet losses, delays, and synchronization may play a fundamental role in this context.

Some key characteristics that influence the performance of the systems in Figs. 1-3 are listed in the following.

- wireless channel variations;

- packet loss probability;

- power consumption;

- communication delays;

- signal quantization;

- node cooperation;

- cross-layer requirements.

We believe that in WSNs with multi-hop communication, these characteristics should be simultaneously investigated under realistic assumptions. Specifically, joint design of communication and control with a cross-layer methodology seems necessary [10]. This approach is motivated by the interdependence of the different communication parameters and the control algorithm performance.

In the next sections, we provide some examples for each of the configurations above, and discuss how they can be included in the design framework.

\section{DISTRIBUTED SOURCE CODING AND PACKET AGGREGATION}

One of the enabling technologies for WSNs is Distributed Source Coding (DSC). DSC refers to a closed-loop distributed control algorithm for the compression of multiple correlated sensor outputs that do not communicate with each other. The compressed outputs are sent to a gathering node for joint decoding.

The Slepian-Wolf Theorem [11] is an encouraging conceptual basis for DSC. The intuition behind Slepian-Wolf coding has been 
demonstrated in [12], and a DSC using syndromes has been proposed in [13].

A basic remark related to deployment of DSC in realistic environments is that header overheads in assembled data units may significantly reduce the advantages promised by DSC. Indeed, in WSNs multi-hop routing is implemented and each node acts as a router that relays packets received from other nodes and, at the same time, as a source of information. As proposed in [14], a node could aggregate packets coming from other nodes with its own data in order to reduce the impact of packet overhead, thus decreasing the generated traffic. We believe that in WSNs with multi-hop communication, DSC topologies and packet aggregation (PA) should be simultaneously designed under realistic assumptions.

Some recent contributions can be found in the literature that deal separately with either DSC or PA. In [15], four topologies for DSC have been investigated with respect to packet losses and energy efficiency. In [16], the authors have studied the problem of finding the optimal aggregation alternative for a WSN that implements DSC. In [17], the problem of optimizing data gathering according to the data source correlation is investigated. The idea of PA was first introduced in [14], where the authors proposed a framework for routing, data compression, and packet aggregation, whereas in [18] classifications of aggregation protocols for periodic measures have been proposed.

Although the relevant existing contributions (e.g., [14]-[18]) have the merit to include detailed network scenarios in DSC, they neglect some important aspects, such as PA and fragmentation, and overhead reduction. However, the aggregation scheme without packet fragmentation is prohibitive for real multi-hop sensor networks. To the best of our knowledge, no contributions can be found in the literature that address jointly the problem of packet aggregation at packet level and DSC schemes, with realistic models of the packet loss probability and automatic repeat request (ARQ) protocol.

\subsection{System Scenario}

Consider an area over which $N$ nodes are uniformly deployed; they transmit toward a sink that acts as a gathering node. As assumed e.g., in [15], the network topology is organized in a tree, where each node is connected to a parent node (see Fig. 4) and produces a number of measures per snapshot. Each node performs

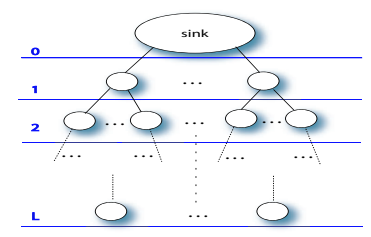

Figure 4: Network topology with $L$ levels.

the following tasks: it encodes the measured data according to a DSC algorithm; then it receives packets from the node children and transmits packets toward the parent node.

The packet frame specified for the Berkeley motes [19] [20] are adopted as a reference. Therefore, a packet is composed as follows: a preamble and a start symbol of $P$ bytes; a data link header of $M$ bytes, a network header of $N$ bytes; a payload of pay $y_{i}$ bits corresponding to the measures of the generic node of the level $i$, where the payload may be variable according to the DSC scheme and aggregation mechanism; $C R C$ bytes for the forward error detection; ID bytes for the node identification; finally, a Manchester coding technique is employed.

\subsection{DSC Schemes}

Let us denote with $Y_{1}, Y_{2}, \ldots, Y_{N}$ the data measured by the nodes $i, \ldots, N$. As it is studied in [17], we consider four DSC coding scheme: No-Slepian-Woolf (NOSW), Sequential SlepianWoolf (SEQ), Slepian-Woolf Clustered (CL), and Slepian-Woolf Master Slave (MS).

In the NOSW scheme, each sensor encodes the measurements independently of other $N-1$ nodes, and with a number of bits given by the entropy of the source $H\left(Y_{i}\right), i=1, \ldots N$. In the SEQ scheme, node 1 performs the coding of $Y_{1}$ with $H\left(Y_{1}\right)$ bits; node 2 perform the coding of $Y_{2}$ with a $H\left(Y_{2} \mid Y_{1}\right)$ bits under the assumption that the decoder knows $Y_{1}$; finally, node $N$ performs the encoding with $H\left(Y_{N} \mid Y_{N-1} \ldots Y_{1}\right)$ bits, under the assumption that the decoder knows $Y_{1}, \ldots, Y_{N-1}$. In the CL case, the nodes are grouped in $K$ clusters, and in each cluster there are $N / K$ nodes. The clusters are formed with the sub-trees that have as root the nodes of level 1 . We assume that in each cluster there is the same number of nodes. For each cluster, a SEQ coding scheme is adopted independently from the other groups and with respect to the common sink of all clusters. In the MS scheme, the nodes are grouped in clusters as in the case of CL, but a master node is selected in each cluster. Moreover, for each node, the DSC is performed with respect to the master and not to the sink.

\subsection{Packet Aggregation}

Three alternatives for packet aggregation can be found in literature [16]: the classic multihop (CMH), where the nodes relays packets without any aggregation; the aggregate multihop (AMH) (see Fig. 5), where the nodes collect the received packets, aggregate them at the MAC layer into a single packet and retransmit it; the Fragmented Aggregate Multihop (FAMH) (see Fig. 6), which is similar to AMH but with the difference that, when an aggregated packet has dimension larger than a threshold, it is fragmented in multiple packets.

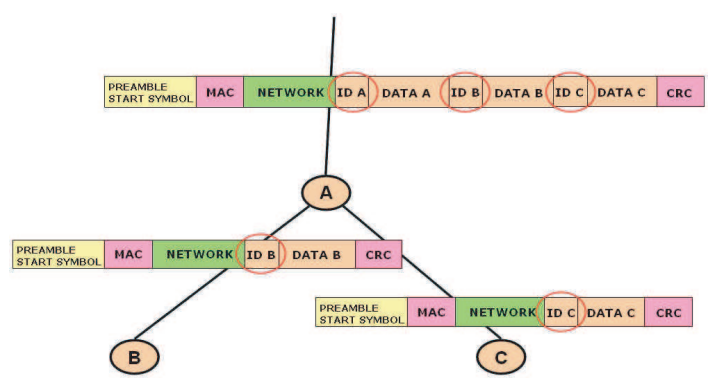

Figure 5: Aggregated Multihop (AMH).

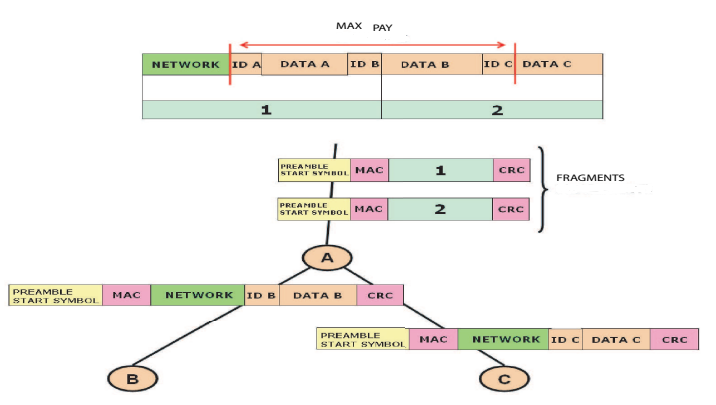

Figure 6: Fragmented Aggregated Multihop (FAMH).

\subsection{Performance Indexes}


Performance analysis is based on the evaluation of the following set of parameters [7]: the average energy consumption of the network $E_{n}$, loss rate $L$, which is the fraction of measurements that cannot be correctly decoded at the gathering node, total coding efficiency for each operative scenario

$$
\eta=1-\frac{E_{n}}{E_{n}(N O S W)},
$$

and coding efficiency for each node

$$
\eta_{i}=1-\frac{B_{T X i}}{B_{T X i}(N O S W)},
$$

where $B_{T X i}$ is the mean number of bytes transmitted by a single $i$-level node. Note that $E_{n}$ and $\eta_{i}$ are dependent on the adopted PA and DSC scheme.

\subsection{Implementation}

A detailed performance evaluation of the packet aggregation mechanisms and DSC was carried out in [7]. Specifically, numerical implementation shows that, in order to obtain significant advantages in terms of energy consumption through the use of DSC, it is important to operate as follows: to use aggregation techniques in order to reduce header overhead; to adopt ARQ protocols, which reduce the loss factor and enhance the coding efficiency, clustered or masterslave coding schemes, because the SEQ is characterized by a larger value of the loss factor.

The optimization of performance indexes when the channel is time-variant and the nodes lack of synchronization is an open issue.

\section{COOPERATIVE DISTRIBUTED DIVER- SITY}

Cooperative diversity is a technique that exploits groups of sensor nodes randomly placed to cooperatively relay a common received signal toward a destination, with the goal to combat severe attenuations or disconnections of signal strength.

Cooperative diversity for signals and applications with disconnection or outage constraints, while minimizing the energy consumption of the sensor nodes, is a relevant issue. In fact, disconnection events have strong influence on the performance of upper layers in the protocol stack, e.g., [21, 22, 8]. The importance of outage events for WSNs was also recognized in [23], where the authors characterized the wireless links for WSNs as connected, transitional and disconnected.

\subsection{System Scenario}

Consider the scenario in Fig. 7: we assume a mobile sensor node, called MS, that broadcasts a signal carrying traffic for a real time application. There is a cluster of $N$ sensor nodes that receive the broadcasted signal and retransmit it to a Sensor Collector (SC), which either has larger computation capabilities, or is directly connected to such a computation resource. The MS could transmit the status information associated to the controlled plant as in [2], where the communication between the actuator at the MS and the controller at the SC employs a WSN that introduces constraints on the loop stability.

There is no direct link between the MS and the SC, the signal has to pass through the relaying nodes that cooperatively weight the received signals to improve the quality of the signal eventually received by the SC. The sensor nodes transmit using different channels, for example they could use two different frequencies, time slots or codes. We assume that the retransmission of signals does not induce appreciable delay.

The links between the MS and the relaying nodes are frequency flat, slowly time variant and affected by path loss, shadow and fast fading. This assumptions are representative of the Industrial, Scientific, and Medical (ISM) transceivers, which are commonly em- ployed in WSNs [24], [25]. We assume the relay sensor nodes and the SC fixed and in visibility. Consequently, the channel coefficients describing the links between them are assumed known with a good approximation. This set up is basically equivalent to the one considered in [26].

The channel gain between the moving sensor node and the generic node $i$, at a given time $n$, is denoted with

$$
h_{i}(n)=g_{i}(n) \sqrt{L_{i}(n) P_{L}\left(d_{i}\right)},
$$

and is comprehensive of path-loss $\left(P_{L}\left(d_{i}\right)\right.$, computed at the distance $\left.d_{i}\right)$, shadowing $\left(L_{i}(n)\right.$, having standard deviation, in $d B$, $\left.\sigma_{L_{i}}\right)$ and fast fading $\left(g_{i}(n)=e^{j \phi_{i}(n)}\right.$, having standard deviation $\sigma_{g_{i}}$, where $\phi_{i}(n)$ is the fast fading phase). The generic node $i$ is not continuously awake, but may be in a sleep condition. The activity state of the node is modelled with the binary random variable $\nu_{i}(n)$, with $P\left[\nu_{i}(t)=1\right]=\mu_{i}$ and $P\left[\nu_{i}(t)=0\right]=1-\mu_{i}$, where $\mu_{i}$ is said to be the activity factor (or duty-cycle) of the node.

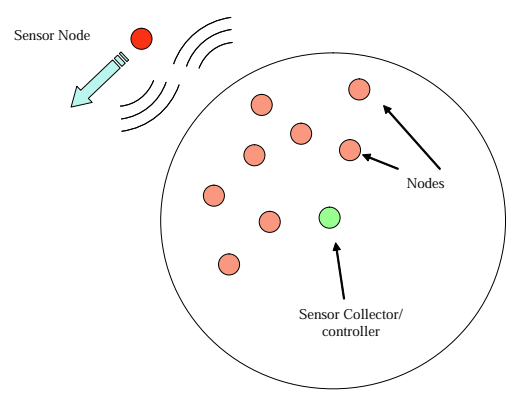

Figure 7: System overview of the WSNs with Cooperative Diversity.

The SC receives simultaneously the retransmitted signals from the various sensor nodes, and therefore performs the sum of the signals. The complex envelope of the received signal is, hence, expressed as follows:

$$
y(n)=\sum_{i=1}^{N} w_{i}(n) \nu_{i}(n)\left[h_{i}(n) s(n)+\varepsilon_{i}(n)\right]+\varepsilon(n),
$$

where $w_{i}(n)=\sqrt{P_{i}(n)} e^{-j \phi_{i}(n)}, i=1, \ldots, N$ are the complex values of the node weighting coefficients, and for the sake of notation simplicity, we include in the node power $P_{i}(n)$ also the known channel gains between the nodes and the SC; $\varepsilon_{i}(n)$ is the noise component at node $i$, and is assumed as white Gaussian noise (AWGN), with power spectral density given by $\sigma_{i}^{2}$; finally, $\varepsilon(n)$ includes the interferences and AWGN noise at the receiver input of the SC, with power spectral density $\sigma_{d}^{2}$.

We denote the power coefficient with the vector $\mathbf{P}(n)=\left[P_{1}(n)\right.$, $\left.P_{2}(n), \ldots, P_{N}(n)\right]$, the fading coefficients with the vector $\boldsymbol{h}(n)=$ $\left[h_{1}(n), h_{2}(n), \ldots, h_{N}(n)\right]$ and the activity factors with the vector $\boldsymbol{\nu}(n)=\left[\nu_{1}(n), \nu_{2}(n), \ldots, \nu_{N}(n)\right]$.

The Signal to Interference + Noise Ratio (SINR) corresponding to (4) is expressed as follows [27]:

$$
\operatorname{SINR}(\boldsymbol{P}, \boldsymbol{h}, \boldsymbol{\nu}, n)=\frac{E_{s} \sum_{i=1}^{N} P_{i}(n) \nu_{i}(n) r_{i}(n)}{\sum_{i=1}^{N} P_{i}(n) \nu_{i}(n) \sigma_{i}+\sigma_{d}},
$$

where $E_{s}$ is the energy of the transmitted signal, while $r_{i}(t)=$ $\left|h_{i}(n)\right|^{2}$. Let us note that dependence of the SINR on the powers of the relaying nodes, the channel gains and the activity factors has been evidenced. Moreover, note that (5) is a time variant stochastic process since the fading is assumed to be time variant. 
The signal (4) is said to be in outage or disconnected if the SINR (5) falls below a minimum quality threshold $\gamma$. In particular, the probability of the outage events is defined as follows:

$$
P_{\text {out }}=P[\operatorname{SINR}(\boldsymbol{P}, \boldsymbol{h}, \boldsymbol{\nu}, n)<\gamma] .
$$

\subsection{Power Minimization}

Let us denote by $\bar{P}_{\text {out }}$ the maximum allowed value of the outage probability. The minimization of the transmission powers of the sensors, under the constraint on the outage probability becomes:

$$
\left\{\begin{array}{cl}
\min _{\mathbf{P}(\mathbf{n})} & \sum_{i=1}^{N} \rho_{i} \mu_{i} P_{i}(n) \\
& \\
\text { s.t. } & P[S I N R(\boldsymbol{P}, \boldsymbol{h}, \boldsymbol{\nu}, n)<\gamma]<\bar{P}_{\text {out }} \\
& P_{\text {min }} \leq P_{i} \leq P_{\text {max }} i=1, \ldots, N
\end{array}\right.
$$

where $P_{\min }$ and $P_{\max }$ are the minimum and maximum values allowed for the power coefficient; $\rho_{i} \in[0,1] i=1, \ldots, N$ is an adaptive constant that represents the residual energy of the relay nodes.

\subsection{Implementation}

The solution of the optimization problem requires firstly explicit derivation of the outage probability (see [8] and [28] for details), and then the estimation of the fading parameters by the nodes, that subsequently retransmit them to the SC. Note that it is not required to achieve the deterministic knowledge of the fading coefficients for computation of the outage probability, but only the estimation of the first and second order moments. Consequently, the cost of the estimation operations is computationally reduced and, hence, suitable for sensor nodes.

Numerical implementation of the cooperative distributed diversity has been carried out. We have considered the same scenario adopted in [23]. Specifically, we assume that the MS transmits with a power of $0 \mathrm{~dB} B_{m}$, and a reference path loss of $-55 \mathrm{~dB}$. The power spectral density of the noise for the relay nodes is assumed $\sigma_{i}=-115 d B_{m} / M H z$, for $i=1, \ldots, N$, and the same value is set for the SC. The relay powers are assumed to lay in the interval $-30 d B, \ldots, 0 d B$. We further assume $\gamma=7.6 d B, \bar{P}_{\text {out }}=0.01$, and $\rho_{i}=1.0$. We consider wireless scenarios where the optimization problem (7) has been solved for different numbers of nodes (3, 5 , and 8$)$ and different values of the node activity factors $(0.3,0.5$ and 0.9 ). Without loss of generality, nodes are set to the same activity factor. The relay nodes are randomly placed between 5 and 10 meters from the MS and are assumed to experience the same standard deviation of the shadowing $\sigma_{L_{i}}=4$ and the same standard deviation of the fast fading $\sigma_{g_{i}}=0.1$.

In Figure 8, the value of the optimized objective function is reported. As the number of nodes increases, the total power consumption increases in the cases $\mu_{i}=0.5$ and 0.9 , while the case 0.3 exhibits an interesting decreasing. This case is the most relevant since the relay nodes experience a low duty cycle and, at the same time, they are perfectly able to guarantee the performance imposed by the constraints.

\section{COOPERATIVE DISTRIBUTED LOCAL- IZATION}

Sensing and controlling the environment often implicitly assumes that measurements of physical parameters are associated to the position of sensing devices. Moreover, various components of the protocol stack (e.g., routing and MAC algorithms) may benefit from location information of network nodes. Cooperative and Distributed Localization is an example of distributed filtering, where the nodes of a WSNs have to cooperate for defining their position. In

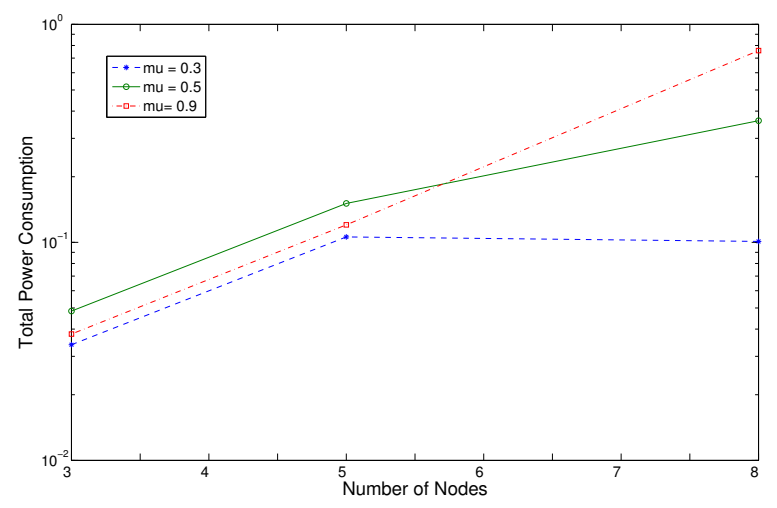

Figure 8: Power consumption $(d B / 10)$ for the first scenario and three cases of the activity factor as function of the number of relay nodes.

such a case, the control algorithm is totally distributed. The key idea of this method is that the two nodes collaborate in order to estimate the relative distance.

\subsection{System Scenario}

The distributed localization algorithm starts with a cooperative ranging phase. One of the nodes acts as master and initiates the ranging transaction by sending a packet to the other node, which acts as a slave by sending back (after a predetermined time interval) the received packet. The master node, through the measurement of the transmit/receive time interval, and the knowledge of the further time interval introduced by the slave node can, at least in principle, estimate the distance between the two nodes. Actually, the lossy nature of the wireless channel often requires a repeated application of the above procedure. However, the method strongly depends on the time resolution capability of the involved nodes.

We assume here that the network consists of $N$ nodes, where each node has a position in $\mathbb{R}^{3}$ specified by $p_{i}=\left(x_{i}, y_{i}, z_{i}\right)$, and let $d_{i j}=\left\|p_{i}-p_{j}\right\|$ be the Euclidean distance between node $i$ and $j$. We assume that a node $i$ knows the distance $d_{i j}$ from each neighbor $j$ after the ranging transaction. Let $\nu_{i j}(n)$ be a binary random variable that assumes the value 1 if node $j$ is connected with node $i, 0$ otherwise. As the algorithm has to be completely distributed, we assume that each node estimates its position by a vector $\hat{p}_{i}$.

\subsection{Localization Refinement}

After a node has got an initial position estimation, it enters a refinement phase, where it iteratively refines this estimation through some algorithm until a stop condition is reached. According to [9], the Steepest Descent Algorithm is used to refine position estimations by minimizing the error functional (Eq. 8), that is the square of the difference between measured distance $\widehat{d}_{i j}$ (in the ranging phase) and estimated Euclidean distance $d_{i j}$ (based on the position estimation information):

$$
\begin{gathered}
F_{i}(n)=\sum_{j=1}^{N}\left[d_{i j}(n)-\widehat{d}_{i j}(n)\right]^{2} \nu_{i j}(n), \\
x_{i}(n+1)=x_{i}(n)+\frac{1}{2} \mu\left[-\frac{\partial F_{i}(n)}{\partial x_{i}}\right] \\
y_{i}(n+1)=y_{i}(n)+\frac{1}{2} \mu\left[-\frac{\partial F_{i}(n)}{\partial y_{i}}\right]
\end{gathered}
$$


where

$$
\begin{aligned}
& \frac{\partial F_{i}(n)}{\partial x_{i}}=2 \sum_{j=1}^{N} \frac{\widehat{d}_{i j}(n)-d_{i j}(n)}{\widehat{d}_{i j}(n)} \cdot\left[x_{i}(n)-x_{j}(n)\right] \nu_{i j}(n) \\
& \frac{\partial F_{i}(n)}{\partial y_{i}}=2 \sum_{j=1}^{N} \frac{\widehat{d}_{i j}(n)-d_{i j}(n)}{\widehat{d}_{i j}(n)} \cdot\left[y_{i}(n)-y_{j}(n)\right] \nu_{i j}(n)
\end{aligned}
$$

where $\left(x_{i}(n), y_{i}(n)\right)$ denotes the position at time $n$ and $\mu$ the socalled learning speed.

\subsection{Implementation}

Numerical implementation of the cooperative distributed localization was carried out in [9]. Numerical results show that the algorithm can provide a satisfactory accuracy, but due to the distributed nature of the algorithm, propagation of errors may occur and represents one of the major limitations. How to design controllers which take into account the error propagation remains an open issue which is currently under investigation.

\section{CONCLUSIONS}

In this paper, a taxonomy of the most relevant configurations of cooperative distributed processing and control over WSNs has been proposed. The goal is to define a common design framework, where cross-layer approach and joint design of communication and control are fundamental aspects. Specifically, we have described three main scenarios where communication and control aspects interact, and we have evidenced the most relevant parameters and characteristics that influence the system design. Illustrative examples of the configurations have been provided in terms of distributed source coding and packet aggregation, collaborative diversity and collaborative localization.

Future work is being focused on the investigation of control methods which are adaptive to the variations of the most relevant parameters in the system. In this context, the analytical tools provided by hybrid control theory seems to be appealing. Specifically, an adaptive hybrid controller for selection of the best configurations of packet aggregation and DSC scheme is desirable when the nodes lack of synchronization and the channel is time variant. The design of a hybrid controller that avoids the blow up of the position estimation in the distributed cooperative localization is an open issue. Similarly, hybrid controllers for the selection of the nodes in cooperative diversity deserve attention.

\section{REFERENCES}

[1] A. Nosratinia, T. E. Hunter, A. Hedayat. Cooperative communication in wireless networks. IEEE Communication Magazine, October 2004.

[2] X. Liu, A. Goldsmith. Wireless network design for distributed control. IEEE Conf. on decision and control, June 2004.

[3] P. Ögren, M. Egerstedt, and X. Hu. A control Lyapunov function approach to multi-agent coordination. IEEE Trans. on Robotics and Automation, 18(5):847-851, 2002.

[4] X. Liu, A. Goldsmith. Cross-layer design of distributed control over wireless network. Systems and Control: Foundations and Applications, (Ed. T. Basar), Birkhauser, 2005.

[5] A. Giridhar, P. R. Kumar. Towards a theory of in-network computation in wireless sensor networks. IEEE Wireless Comm. Magazine, submitted, 2005.

[6] K. H. Johannson, F. Santucci, editors. Report on distributed control over wireless network. NoE HYCON (HYbrid CONtrol: Taming Heterogeneity and Complexity of Networked Embedded Systems, Network of Excellence, European Commission, IST program, FP6), 2005.
[7] L. Di Paolo, C. Fischione, F. Graziosi, F. Santucci, S. Tennina. Performance evaluation of distributed source coding and packet aggregation for wireless sensor networks. submitted to IEEE Globecom 2006, 2006.

[8] C. Fischione, A. Bonivento, A. Sangiovanni-Vincentelli, F. Santucci, K. H. Johansson. Performance analysis of collaborative spatio-temporal processing for wireless sensor networks. IEEE CCNC 2006, January 2006.

[9] G. Di Stefano, F. Graziosi and F. Santucci. Distributed positioning algorithm for ad-hoc networks. Int. Workshop on Ultra Wideband Systems (IWUWBS), Oulu, 2003.

[10] M.D. Di Benedetto, A. DInnocenzo, G. Pola, C. Rinaldi, F. Santucci. Modeling of adaptive behaviours in control over wireless networks. Invited session on "distributed decision-making over ad-hoc networks", 17th MTNS, 2006.

[11] D. Slepian and J.K. Wolf. Noiseless coding of correlated information sources. IEEE Trans. Inform. Theory, vol. 19, pp. 471-480, July 1973.

[12] S. Pradhan, J. Chou, and K. Ramchandran. Duality between source coding and channel coding and its extension to the side information case. IEEE Trans. Inform. Theory, vol. 49, pp. 1181-1203, May 2003.

[13] S. Pradhan and K. Ramchandran. Distributed source coding using syndromes (discus): Design and construction. IEEE Trans. Inform. Theory, vol. 49, pp. 626-643, March 2003.

[14] D. Petrovic, R. C. Shah, K. Ramchandran, J. Rabaey. Data funneling: Routing with aggregation and compression for wireless sensor networks. IEEE SNPA, 2003.

[15] D. Marco, D. L. Neuhoff. Reliabiity vs. efficiency in distributed source coding for field-gathering sensor networks. IEEE IPSN 04, 2004.

[16] Sueng Jun Baek, G. de Veciana, Xun Su. Minimizing energy consumption in large-scale sensor networks through distributed data compression and hierarchical aggregation. IEEE Journal on Selected Areas in Communications, 2004

[17] R. Cristescu, B. Beferull-Lozano, M. Vetterli. On network correlated data gathering. IEEE INFOCOM, 2004.

[18] U. Roedig, A. Barroso, C. J. Sreenan. Determination of aggregation points in wireless sensor networks. IEEE EUROMICRO, 2004.

[19] CrossBow Technologies. http://www.xbow.com.

[20] TinyOS. http://webs.cs.berkeley.edu/tos/.

[21] M. Zorzi. Outage and error events in bursty channels. IEEE Trans. on Comm., Vol. 46, no.3, 1998.

[22] L. Yang, M. S. Alouini. On the average outage rate and average outage duration of wireless communication systems with multiple cochannel interferers. IEEE Trans. on Wir. Comm., Vol. 3, N. 4, July 2004.

[23] M. Zuniga, B. Krishnamachari. Analyzing the transitional region in low power wireless links. SECON, 2004.

[24] H. Karvonen, Z. Shelby, C. Pomalaza-Rez. Coding for energy efficient wireless embedded networks. IWWAN, 2004.

[25] J. Polastre, R. Szewczyk, D. Culler, "Telos: Enabling Ultra-Low Power Wireless Research. Telos: Enabling ultra-low power wireless research. IPSN/SPOTS, April, 2005.

[26] R. Mudumbai, J. Hespanha, U. Madhow, G. Barriac. Scalable feedback control for distributed beamforming in sensor networks. IEEE Int. Symposium on Inf. Theory, 2005.

[27] G. L. Stüber. Principles of Mobile Communication. Kluwer Academic Publishers, 1996.

[28] C. Fischione, F. Graziosi, F. Santucci. Approximation of a sum of onoff lognormal processes with wireless applications. IEEE ICC, 2004. 